\title{
SOBRE O QUE FALAMOS QUANDO FALAMOS EM GÊNERO NA CIÊNCIA DA INFORMAÇÃO?
}

\author{
¿SOBRE QUÉ HABLAMOS CUANDO HABLAMOS DE \\ GÉNERO EN LA CIENCIA DE LA INFORMACIÓN?
}

\author{
Mell Siciliano* \\ Cleiton da Mota de Souza** \\ Clara de Mello e Souza Meth***
}

\begin{abstract}
Resumo
Introdução: Atualmente diversos campos conduzem pesquisas sobre o domínio "gênero". Este estudo investiga sob quais perspectivas este domínio é abordado pela Ciência da Informação $(\mathrm{Cl})$. Objetivo: O objetivo é identificar como o campo se apropria e/ou contribui para os estudos sobre gênero; tendo como norteadora a acepção de que gênero é um termo relacionado aos papéis sociais e expectativas de comportamento atribuídas as pessoas em função do seu sexo. Metodologia: Foi realizada coleta e análise das palavras-chave de 588 artigos indexados na Library and Information Science Abstracts (LISA), entre 1980 e 2016. Considerando o grande número de palavras-chave recuperadas, foi gerada uma nuvem de tags; permitindo a identificação dos termos mais utilizados por década. Resultados: Os resultados indicam que o domínio "gênero" foi abordado pela $\mathrm{Cl}$ sob diferentes perspectivas ao longo dos anos, mas mantendo, de maneira geral, o foco principal em questões relacionadas a estudos sobre mulheres e diferenças de gênero. Observa-se também que o domínio é tratado pela $\mathrm{Cl}$ principalmente dentro de tópicos tradicionais da área, como Recuperação da Informação e Comunicação Científica. Conclusões: Ressaltase que o panorama resultante deste estudo abre novas possibilidades de investigação sobre como a $\mathrm{Cl}$ aborda o domínio "gênero" em suas pesquisas científicas.
\end{abstract}

Palavras-chave: Gênero. Ciência da Informação. Estado de Conhecimento.

\footnotetext{
* Mestranda do Programa de Pós-Graduação em Ciência da Informação convênio Instituto Brasileiro de Informação em Ciência e Tecnologia e Universidade Federal do Rio de Janeiro. Email: mellsiciliano@gmail.com.

** Mestrando do Programa de Pós-Graduação em Ciência da Informação convênio Instituto Brasileiro de Informação em Ciência e Tecnologia e Universidade Federal do Rio de Janeiro. Email: cleiton.mota.s@gmail.com.

*** Mestranda do Programa de Pós-Graduação em Ciência da Informação convênio Instituto Brasileiro de Informação em Ciência e Tecnologia e Universidade Federal do Rio de Janeiro. Email: claramsm@uol.com.br.
}

Inf. Inf., Londrina, v. 22, n. 2, p. 144 - 165, maio/ago., 2017.

http:www.uel.br/revistas/informacao/ 


\section{INTRODUÇÃO}

O domínio "gênero" perpassa, atualmente, várias esferas de discussão na sociedade. Isso acontece pois na medida em que novas reflexões sobre gênero são consideradas, a necessidade de novos e mais profundos debates se torna evidente. As reflexões sobre gênero geram - ou deveriam gerar novas adaptações em todos os âmbitos sociais. Tais adaptações precisam ser consideradas, debatidas e aprofundadas.

A ciência reflete sobre gênero, na sua moderna acepção, desde o final dos anos 1960. O domínio está tradicionalmente atrelado às pesquisas em Ciências Sociais, sendo abordado pela Sociologia, Psicologia, Educação, entre outros. Entretanto, por ser um campo interdisciplinar, a Ciência da Informação (CI) também reflete sobre gênero.

Ao considerar "gênero" como domínio, é importante ter em mente a dificuldade em organizar o conhecimento, como nos lembra Hjørland (2008), principalmente pela inexistência de uma teoria unificada de organização do conhecimento. Como gênero é um conceito atrelado tradicionalmente a outros campos, é natural que sua posição na teia de conhecimento destes campos seja mais consolidada do que na $\mathrm{Cl}$. É necessário, portanto, refletir sobre o domínio "gênero" na $\mathrm{Cl}$, no âmbito da organização do conhecimento, com fins de identificar como o campo se apropria e/ou contribui para os estudos relacionados a gênero.

A contribuição se dará pela abordagem do Estado de Conhecimento, considerando que Estado de Conhecimento é "identificação, registro, categorização que levem à reflexão e síntese sobre a produção científica de uma determinada área, em um determinado espaço de tempo" (MOROSINI; FERNANDES, 2014); tendo também como enfoque a presença do novo na produção científica (o novo pode ser uma nova abordagem, análise, tópico, entre tantos outros).

Inf. Inf., Londrina, v. 22, n. 2, p. 144 - 165, maio/ago., 2017. http:www.uel.br/revistas/informacao/ 


\section{O DOMÍNIO “GÊNERO”: ALGUMAS CONSIDERAÇÕES}

Definir um termo é uma tarefa inglória. As palavras carregam conceitos, e estes conceitos, por sua vez, estão em constante construção.

O conceito moderno do termo gênero surgiu no final dos anos 1960, quando o psiquiatra Robert Stoller - ao pesquisar meninos e meninas que, por causa de problemas anatômicos, foram criados como se fossem do sexo oposto - estabeleceu a diferença entre sexo e gênero. A partir desta pesquisa, publicada no livro "Sex and gender: on the development of masculinity and femininity", Stoller percebeu que existiam duas categorias: uma biológica, que demonstraria que o ser humano, como espécie, precisa de diferenciação sexual para se reproduzir (sexo), e outra psicológica, que seriam os comportamentos atribuídos e esperados de cada sexo (gênero). Essa acepção do termo gênero começou a se disseminar na academia a partir dos anos 1980 (MATOS, 2015).

Sendo assim, o termo gênero não é sinônimo do termo sexo, e não corresponde, portanto, às diferenças biológicas dos indivíduos. Gênero é um termo representativo, que está relacionado aos papéis sociais e às expectativas de comportamento atribuídas às pessoas em função de seu sexo biológico. Para Scott (1986), gênero se refere à organização social da diferença sexual. Sendo assim, as questões de gênero perpassam por várias esferas da sociedade, e englobam tantos outros conceitos e questões, como, por exemplo, igualdade e identidade de gênero, identidade sexual, feminismo e machismo, orientação sexual, entre outros.

É importante ressaltar, que mesmo o conceito moderno de gênero está sendo revisto e reconstruído. Alguns autores salientam a importância de desconectar o conceito de sexo do conceito de gênero. Sob tal perspectiva, os debates sobre relações de gênero cedem espaço para debates sobre identidades de gênero (FLEURY-TEIXEIRA; CHIMELI, 2015).

A Ciência da Informação também se debruçou sobre o domínio "gênero". Alguns estudos publicados no Brasil, como os de Espírito Santo (2008), Bufrem e Nascimento (2012) e Dias e Lima (2013) tinham como objetivo investigar de 
que maneira o domínio "gênero" é trabalhado pela Cl. Todos estes estudos, entretanto, tiveram como corpus de análise somente artigos indexados pela Base de Dados Referenciais de Artigos de Periódicos em Ciência da Informação (BRAPCl), tendo como foco, portanto, a produção científica brasileira.

Entretanto, mesmo com o foco em publicações brasileiras, os resultados encontrados por Espírito Santo (2008), Bufrem e Nascimento (2012) e Dias e Lima (2013) fornecem um panorama de como a Ciência da Informação aborda o domínio "gênero".

Espírito Santo (2008) relata que apenas 18 artigos relacionados a este domínio - a autora focou no assunto "desigualdade de gênero" - foram publicados em revistas nacionais e internacionais indexadas no Portal de Periódicos da Capes, e também em edições do Enancib, entre 2000 e 2007. Este estudo aponta que o item mais explorado no assunto "desigualdade de gênero" compreende a discrepância de artigos publicados por homens e mulheres (produtividade na academia); outros artigos abordam questões relacionadas à indexação, linguística e "acesso à informação e à tecnologia da informação e seu uso por mulheres" (ESPÍRITO SANTO, 2008).

Segundo Bufrem e Nascimento (2012), o domínio é pouco explorado pela Cl no Brasil. Já Dias e Lima (2013), constatam a ausência de publicações relacionadas a questões de gênero e sobre mulheres em periódicos de Ciência da Informação brasileiros indexados na BRAPCI durante o período entre 1970 e 1989.

\section{METODOLOGIA}

O presente estudo utilizou dados obtidos na base Library and Information Science Abstracts (LISA). Fundada em 1969, a LISA é uma base de dados referencial que indexa 487 periódicos do mundo inteiro, em diversas línguas, nas áreas de Biblioteconomia e Ciência da Informação. Mantida atualmente pela ProQuest (2016), a LISA é atualizada mensalmente, com mais de mil registros adicionados por atualização. O público-alvo da base inclui 
Mell Siciliano, Cleiton da Mota de Souza, Clara de Mello e Souza Meth

Sobre o que falamos quando falamos em gênero na ciência da informação?

acadêmicos, alunos, pesquisadores, bibliotecários e profissionais de áreas correlatas. No Brasil, é possível acessar a LISA através do Portal de Periódicos da Capes.

A obtenção dos dados foi feita através de busca na LISA com o uso do termo Gender no campo Subject Heading (Assunto Principal) e tipo de documento Journal Article. A escolha pelo tipo de documento visou restringir o resultado a produções científicas da área, excluindo, portanto, resenhas de livro, entrevistas, entre outros. Foram recuperados 588 artigos, entre os anos 1980 e 2016. Não foram recuperados artigos com a palavra-chave Gender antes dos anos 1980. A coleta dos artigos foi feita no dia 31 de janeiro de 2017.

Foram extraídas, para a análise dos assuntos abordados, as palavraschave dos 588 artigos recuperados. Com o objetivo de visualizar possíveis evoluções e mudanças de enfoque temático, os dados foram divididos em décadas, a saber: 1980, 1990, 2000 e 2010. A década de 2010 contém artigos de 2010 a 2016, visto que esta ainda não é uma década completa.

Considerando o grande número de palavras-chave, optou-se pela análise dos termos através da visualização em nuvens de tags; esse tipo de visualização conta a frequência com que as palavras aparecem em um determinado texto. $\mathrm{O}$ tamanho de cada palavra na nuvem está relacionado com a quantidade de vezes em que ela aparece, logo, quanto maior a palavra, maior a sua frequência em determinado texto.

Após a extração foram gerados quatro arquivos - um por década contendo as palavras-chave. Com o uso do programa Microsoft Excel foi feita uma padronização dos termos:

a) como a preocupação do atual estudo é com os temas abordados pela $\mathrm{Cl}$ dentro do domínio "gênero", foram excluídos nomes de lugares e instituições;

b) todas as palavras que apareciam no singular e no plural foram normalizadas para o plural (por exemplo, no caso dos termos Library e Libraries como palavras-chave, o termo no singular foi alterado para o plural);

c) palavras que tem grafia diferente no inglês americano e no inglês britânico foram mescladas, prevalecendo a grafia daquela com maior frequência (por exemplo, behavior e behaviour);

Inf. Inf., Londrina, v. 22, n. 2, p. 144 - 165, maio/ago., 2017. 
d) tendo em vista que as nuvens de tags contam a frequência de palavras e não de expressões, optou-se também por colocar juntas, manualmente, as expressões que apareciam como palavras-chave (ou seja, Gender Differences virou Gender Differences) de modo que elas pudessem ser contadas como uma única palavra pelo gerador de nuvens de tags; isto foi feito pois entende-se que elas carregam um significado que se perderia caso fossem consideradas separadamente.

Cada arquivo foi então inserido separadamente no wordart.com, um site de visualização de nuvem de tags; este site foi escolhido pois remove automaticamente números e palavras que não representam conteúdo, como artigos e preposições. Além disso permite que um grande número de edições de layout sejam feitas na nuvem de tags.

\section{RESULTADOS}

Tendo em vista o grande número de termos recuperados, não foi possível considerar na análise todas as palavras-chave. Em apêndice, a lista completa foi apresentada por décadas (a saber, APÊNDICE A, 1980; APÊNDICE B, 1990; APÊNDICE C, 2000; APÊNDICE D, 2010), para revisão e quaisquer dúvidas e cotejamentos. A título de recorte do corpus, em todos os anos foram analisadas somente os cinco termos e/ou expressões com maior frequência.

Os primeiros artigos encontrados datam da década de 1980, estando, portanto, em consonância com a afirmativa de Matos (2015), quando diz que o interesse pelo domínio se disseminou na academia a partir desta década. Desde então, o crescimento da discussão sobre gênero em Ciência da Informação é evidente.

\subsection{Primeira década: 1980}

$\mathrm{Na}$ década de 1980 foram recuperados somente 4 artigos com 23 palavras-chave no total. A Figura 1 ressalta as cinco palavras ou expressões 
que mais aparecem: Gender (3x), Efficiency (2x), Evaluation (2x), Job Performance (2x) e Library Staff (2x).

Figura 1 - Palavras-chave da década de 1980

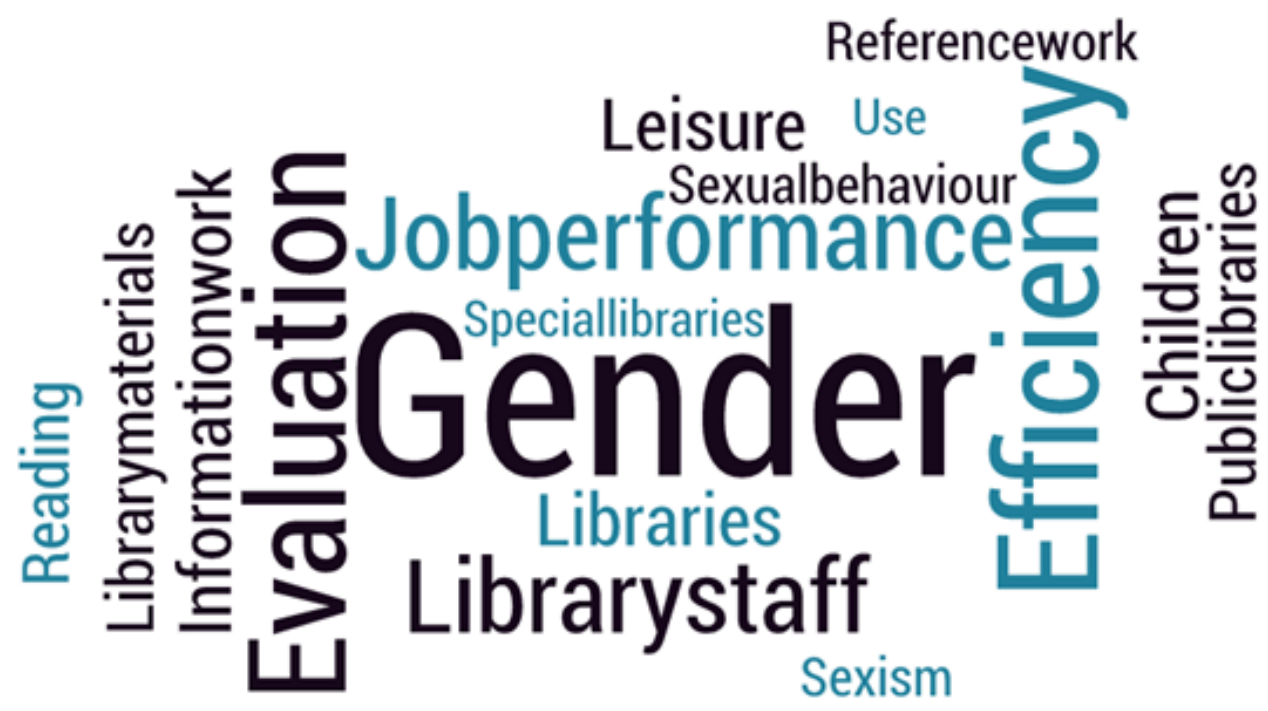

Fonte: Os autores.

São poucos termos e artigos se comparados aos anos subsequentes. Entretanto, é possível inferir pelos termos Library Staff, Job Performance, Efficiency e Evaluation, que o domínio "gênero" aparece ligado à Biblioteconomia e ao trabalho de bibliotecários.

\subsection{Segunda década: 1990}

$\mathrm{Na}$ década de 1990 foram recuperados 78 artigos com 357 palavraschave no total. As cinco palavras ou expressões que mais aparecem são: Gender (59x), Information (12x), Communication (11x), Women (11x), e World Wide Web (10x).

Conforme Figura 2: 
Figura 2 - Palavras-chave da década de 1990.

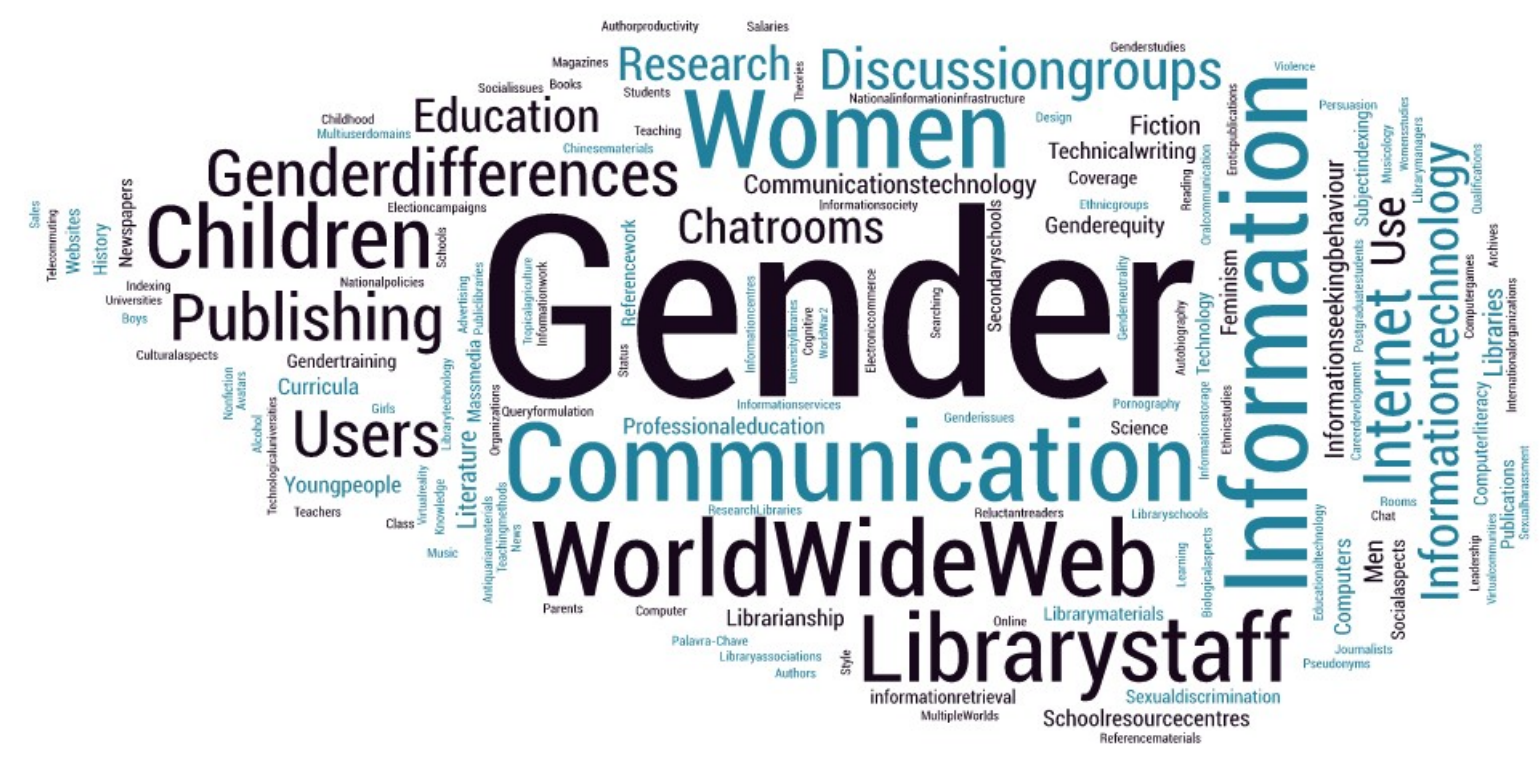

Fonte: Os autores.

As palavras-chave que mais aparecem neste período sugerem uma preocupação maior com atividades relacionadas ao uso da internet, a comunicação e a informação na rede (Information, Communication e World Wide Web). Tal interesse pode ser explicado pelo fato de que a World Wide Web (WWW) surgiu somente em 1989, sendo, portanto, um assunto novo e importante em várias áreas do conhecimento (EUROPEAN ORGANIZATION FOR NUCLEAR RESEARCH, 2013). O aparecimento do termo Women sugere que a preocupação da $\mathrm{Cl}$ estava voltada nesta década para questões relacionada à mulher.

\subsection{Terceira década: 2000}

Na década de 2000 foram recuperados 229 artigos com 1.053 palavraschave no total. As cinco palavras ou expressões que mais aparecem são: Gender (117x), Gender Differences (51x), Women (29x), Information Technology (28x) e Users (27x). Como pode ser visto na Figura 3: 
Figura 3 - Palavras-chave da década de 2000

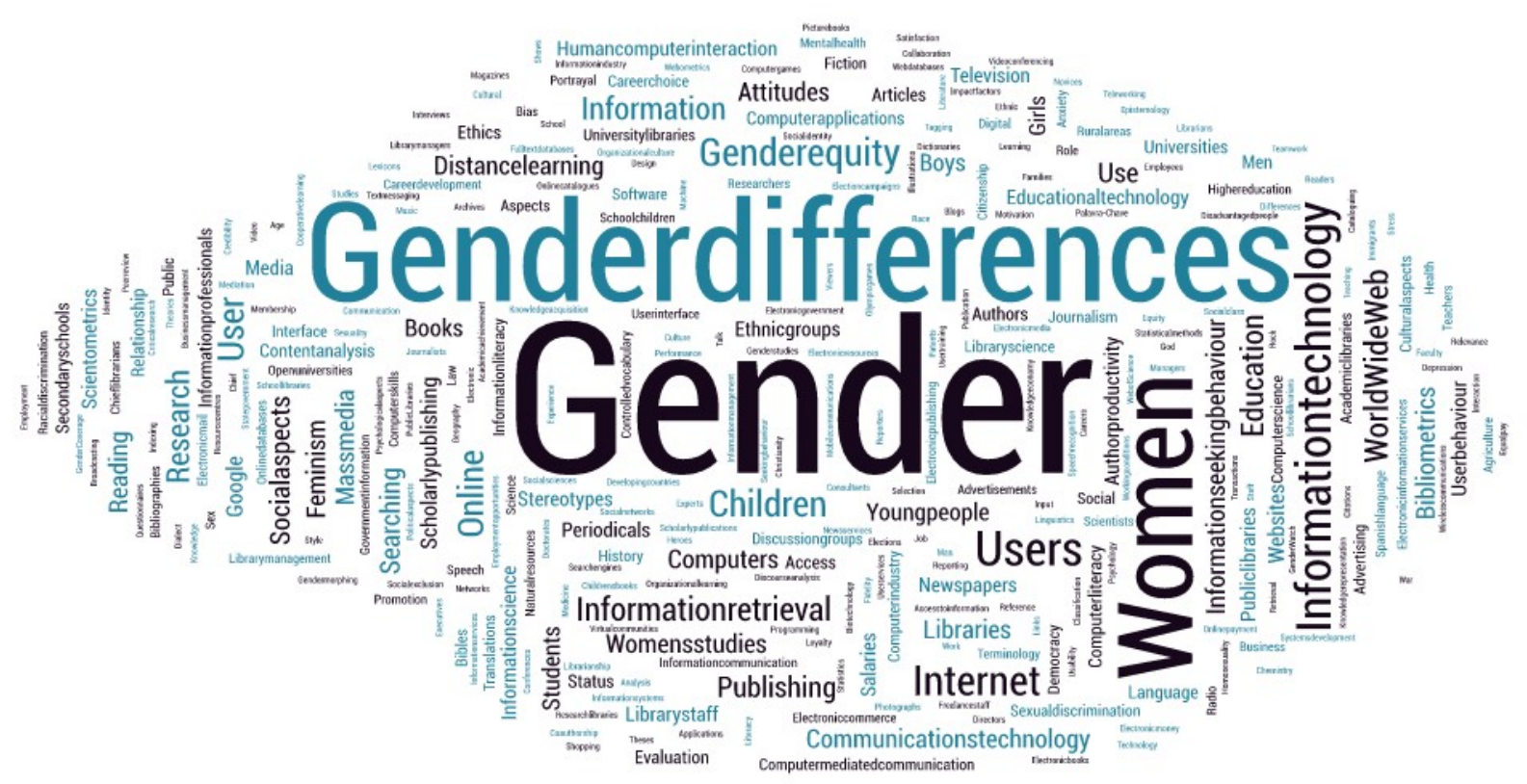

Fonte: Os autores.

Na década de 2000 é possível observar preocupações relacionadas às tecnologias da informação, tendo em vista a frequência de aparecimento do termo Information Technology. A frequência da palavra Users pode sugerir uma preocupação com o uso dessas tecnologias. A palavra Women continua no ranking dos termos mais frequentes, e o aparecimento da expressão Gender Differences entre as palavras-chave mais utilizadas sugere preocupação com os diferentes acessos dados a homens e mulheres em várias esferas da sociedade, como educação, trabalho, entre outros, conforme definição do Tesauro Gender Equality Glossary and Thesaurus, criado pelo European Institute for Gender Equality (EIGE) (EUROPEAN INSTITUTE FOR GENDER EQUALITY, 2016).

\subsection{Quarta década: 2010}

Na década de 2010 foram recuperados 277 artigos com 1.359 palavraschave no total. As cinco palavras ou expressões que mais aparecem são: Gender (145x), Gender Differences (55x), Women (34x), Social Networks (29x) e Scholarly Publishing (23x). Conforme Figura 4: 
Figura 4 - Palavras-chave da década de 2010

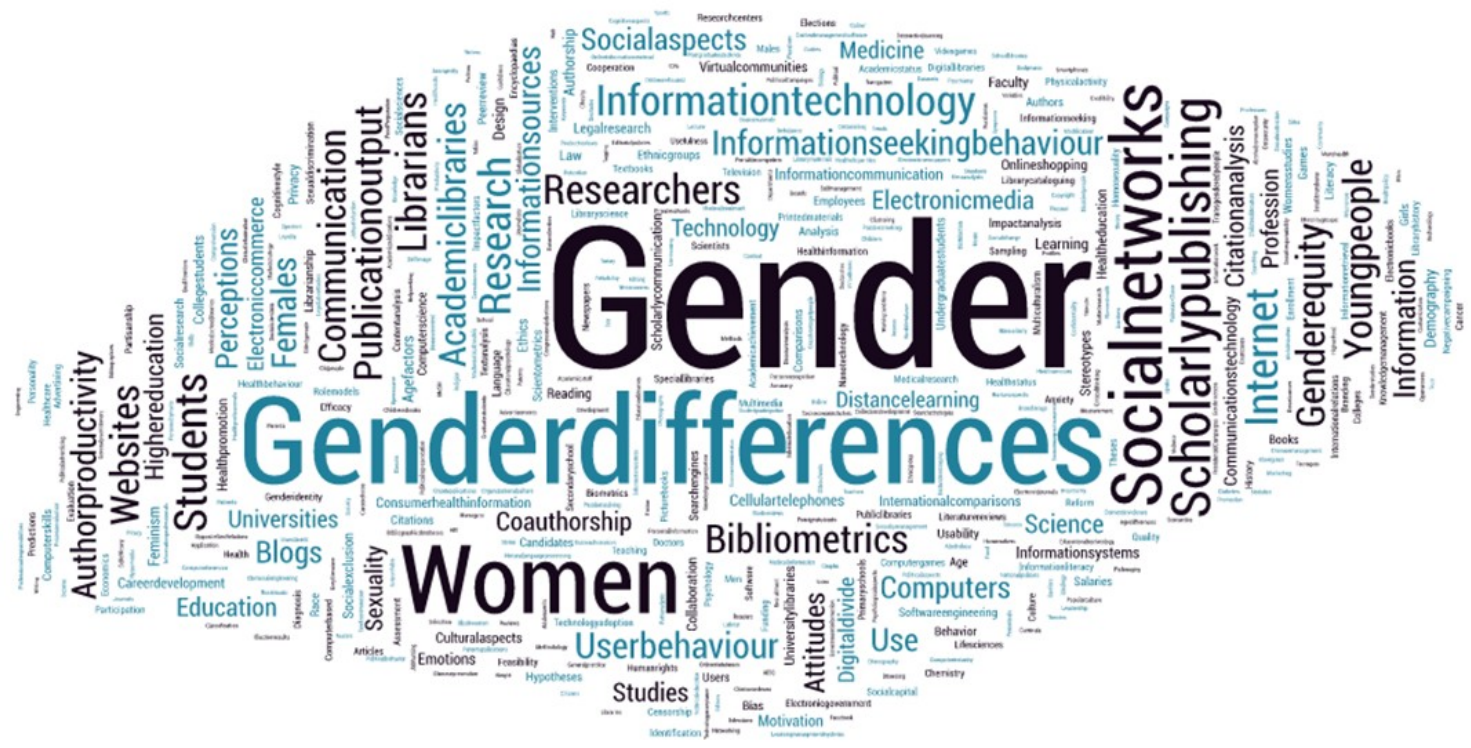

Fonte: Os autores.

Como já sinalizado, a década de 2010 não está finalizada. Os artigos de 2010 a 2016 sugerem que a preocupação com a mulher e as diferenças de gênero continuam, visto a permanência dos termos Women e Gender Differences. A principal novidade é o aparecimento dos termos Social Networks e Scholarly Publishing, que sugerem uma abordagem de gênero voltada para dinâmicas que ocorrem no ambiente acadêmico, como a produtividade de autores e redes de interação entre autores, por exemplo.

\section{DISCUSSÕES}

Observa-se na correlação dos resultados o avanço quanto a estudos que abordam o domínio "gênero" na Ciência da Informação. Como pode ser observado no gráfico a seguir (GRÁFICO 1), ocorre um aumento expressivo no número total de documentos desde os anos 1980 até a atualidade. O gráfico mostra que de 1980 até 2016 o interesse da $\mathrm{Cl}$ sobre gênero cresce de maneira constante. Este crescimento demonstra que as discussões em torno

Inf. Inf., Londrina, v. 22, n. 2, p. 144 - 165, maio/ago., 2017. http:www.uel.br/revistas/informacao/ 
Mell Siciliano, Cleiton da Mota de Souza, Clara de Mello e Souza Meth

do domínio "gênero" ganham cada vez mais espaço nas pesquisas em $\mathrm{Cl}$; a década de 2010, por exemplo, ainda não está completa e já supera a década anterior quanto ao número de artigos publicados sobre gênero.

Gráfico 1 - Artigos sobre gênero indexados na LISA

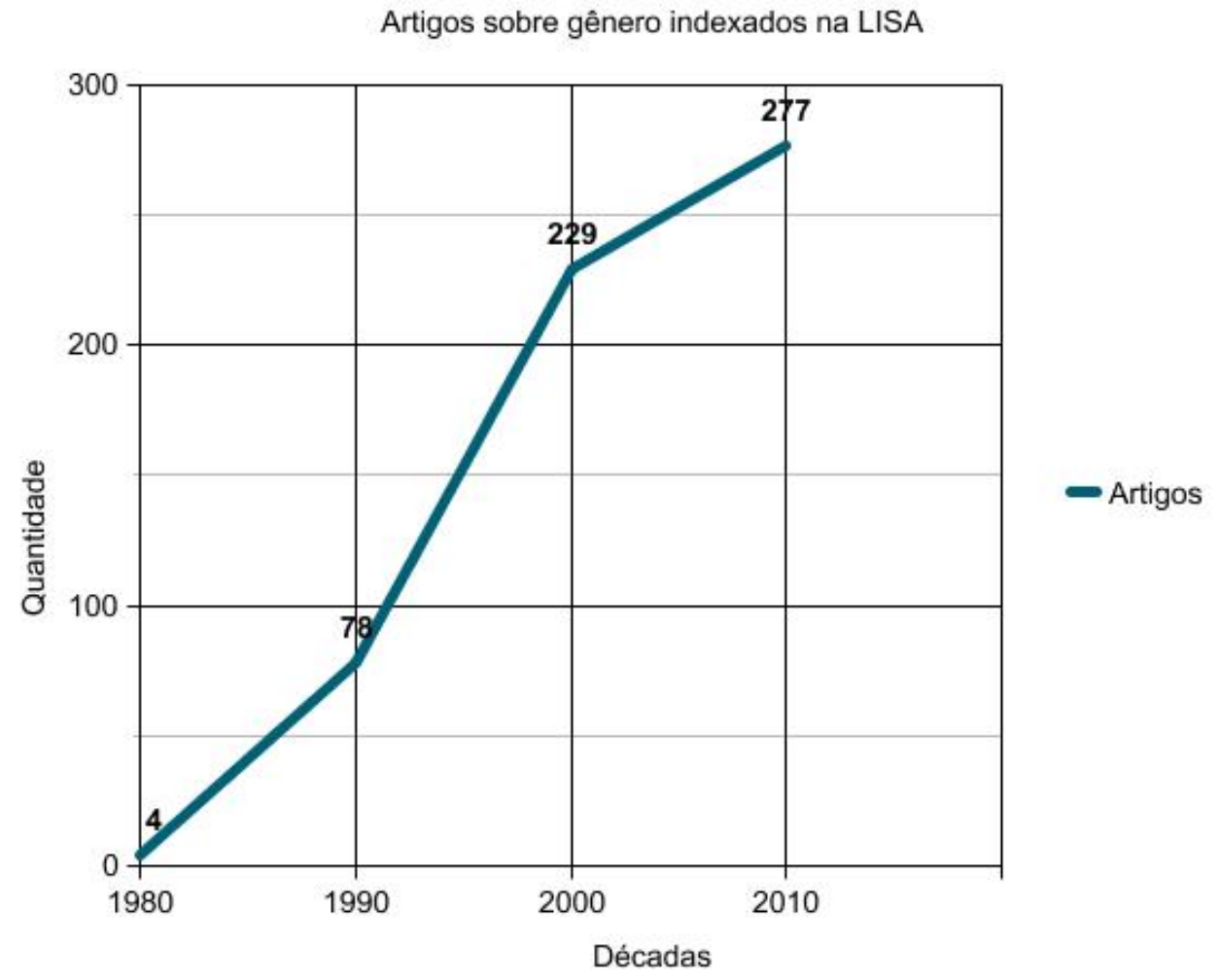

Fonte: Os autores.

É possível especular sobre várias razões para este aumento. O crescimento da discussão sobre gênero em $\mathrm{Cl}$ poderia ser um reflexo, por exemplo, de várias políticas públicas ao redor do mundo, que contribuem para a diminuição das disparidades de gênero, e fomentam as discussões em torno deste domínio. Ainda que não seja possível estipular uma causa exata para o crescente interesse da $\mathrm{Cl}$ sobre gênero, observar a evolução deste interesse é especialmente relevante, tendo em vista que este é um campo com expressiva participação feminina, conforme ressaltado por Bufrem e Nascimento (2012).

Os resultados indicam que tal domínio passa pela $\mathrm{Cl}$ não de maneira central, mas sim dentro de tópicos tradicionais da área, como Recuperação da 
Informação e Comunicação Científica por exemplo. Além disso, a abordagem do domínio "gênero" na $\mathrm{Cl}$ está sensivelmente ligada às questões da mulher e aos debates de diferenças de gênero, com um especial enfoque nos anos 2010 para as dinâmicas ocorridas no ambiente acadêmico. Outras questões como a homossexualidade, por exemplo, aparecem ainda de maneira periférica.

\section{CONSIDERAÇÕES FINAIS}

No presente estudo buscou-se identificar os tópicos mais abordados pela Ciência da Informação dentro do domínio "gênero".

Ainda que a $\mathrm{Cl}$ não traga novas teorias para o domínio "gênero", ela utiliza tais teorias para debater como questões de gênero podem influenciar as diversas esferas tradicionalmente estudadas pela $\mathrm{Cl}$; em vários casos os resultados encontrados podem contribuir para diversas discussões não só na $\mathrm{Cl}$ como também em outros campos. Estudos de produtividade e autoria podem, por exemplo, contribuir para o debate sobre a presença da mulher na academia.

Ademais, retornando ao pensamento de Hjørland (2008) sobre a dificuldade em organizar o conhecimento, a identificação do que a $\mathrm{Cl}$ fala em suas publicações sobre o domínio "gênero" é apenas um primeiro passo para o entendimento do domínio dentro da Cl. Ressalta-se que esta pesquisa, por trazer dados mais amplos, abre um leque de possibilidades para outras análises, como, por exemplo, análises de perfil autoral ou institucional; ou ainda com enfoque em como a $\mathrm{Cl}$ aborda temas específicos dentro do domínio "gênero", como diferenças de gênero ou homossexualidade. Estudos com um caráter mais qualitativo são especialmente recomendados, pois são essenciais para um melhor entendimento de como o domínio é abordado pela Ciência da Informação. 


\section{REFERÊNCIAS}

BUFREM, Leilah Santiago; NASCIMENTO, Bruna Silva do. A questão do gênero na literatura em Ciência da Informação. Em Questão, Porto Alegre, v. 18, n. 3, p. 199-214, dez. 2012. Disponível em: <http://seer.ufrgs.br/index.php/EmQuestao/article/view/33285>. Acesso em: 25 nov. 2016.

DIAS, Karla Cristina Oliveira; LIMA, Francisca Rosimere Alves de. Levantamento das produções sobre mulheres e relações de gênero nos artigos de periódicos em Ciência da Informação. Múltiplos Olhares em Ciência da Informação, [Belo Horizonte], v. 3, n. 2, p. 1-15, out. 2013. Disponível em: <portaldeperiodicos.eci.ufmg.br/index.php/moci/article/view/2166>. Acesso em: 25 nov. 2016.

ESPÍRITO SANTO, Patrícia. Os estudos de gênero da Ciência da Informação. Em Questão, Porto Alegre, v. 14, n. 2, p. 317-332, jul./dez. 2008. Disponível em: $\quad<h t t p: / / s e e r . u f r g s . b r / i n d e x . p h p / E m Q u e s t a o / a r t i c l e / v i e w / 6389 / 4877>$. Acesso em: 25 nov. 2016.

EUROPEAN INSTITUTE FOR GENDER EQUALITY. Gender equality glossary and thesaurus: gender disparities. Lituânia, c2016. Disponível em: <http://eige.europa.eu/rdc/thesaurus/terms/1162>. Acesso em: 25 nov. 2016.

EUROPEAN ORGANIZATION FOR NUCLEAR RESEARCH. The birth of the web. Suíça, 2013. Disponível em: <https://home.cern/topics/birth-web>. Acesso em: 07 dez. 2016.

FLEURY-TEIXEIRA, Elizabeth; CHIMELI, Isabela. Papéis femininos e papéis masculinos. In: FLEURY-TEIXEIRA, Elizabeth; MENEGHEL, Stela N. (Org.). Dicionário feminino da infâmia: acolhimento e diagnóstico de mulheres em situação de violência. Rio de Janeiro: Editora Fiocruz, 2015. p.153-155.

HØRLAND, Birger. What is knowledge organization (KO)? Knowledge Organization, [S.I.], v. 35, n. 2/3, p. 86-101, 2008.

MATOS, Marlise. Gênero. In: FLEURY-TEIXEIRA, Elizabeth; MENEGHEL, Stela N. (Org.). Dicionário feminino da infâmia: acolhimento e diagnóstico de mulheres em situação de violência. Rio de Janeiro: Editora Fiocruz, 2015. p.153-155.

MOROSINI, Marília Costa; FERNANDES, Cleoni Maria Barboza. Estado do Conhecimento: conceitos, finalidades e interlocuções. Educação Por Escrito, Porto Alegre, v. 5, n. 2, p.154-164, jul./dez. 2014. Disponível em: <http://revistaseletronicas.pucrs.br/ojs/index.php/porescrito/article/view/18875>. Acesso em: 25 nov. 2016. 
PROQUEST. Library and Information Science Abstracts (LISA): About. [S.I.], 2016. Disponível em: <http://proquest.libguides.com/lisa>. Acesso em: 20 nov. 2016.

SCOTT, Joan Wallach. Gender: a useful category of historical analysis. The American Historical Review, Oxford, v. 91, n. 5, p.1053-1075, dez. 1986. Disponível em: <http://www-jstor-org.ez29.capes.proxy.ufrj.br/stable/1864376>. Acesso em: 26 dez. 2016.

\title{
Title
}

What do we talk about when we talk about gender in Information Science?

\begin{abstract}
Introduction: Nowadays, various fields conduct research on gender. This study investigates under what perspectives this domain is addressed by Library and Information Science (LIS). Goals: The main goal is to understand how the field appropriates and/or contributes to gender studies; defining gender as a representative term related to social roles and expectations of behavior attributed to people according to their sex. Methodology: Keywords from 588 articles from the International Library and Information Science Abstracts (LISA) were retrieved and analyzed, from 1980 to 2016. Given the large number of keywords retrieved, cloud tags were generated and analyzed in order to identify the most used terms per decade. Results: The results indicate that gender was addressed by LIS from different perspectives over the years, but generally maintained the main focus on issues related to women's studies and gender differences. In addition, it is observed that gender is studied in LIS within traditional topics of the area, such as Information Retrieval and Scientific Communication. Conclusions: This study opens new possibilities for research on how LIS addresses gender in its scientific discoveries.
\end{abstract}

Keywords: Gender. Information Science. State of Knowledge.

\section{Título}

¿Sobre qué hablamos cuando hablamos de género en la ciencia de la información?

\section{Resumen}

Introducción: Actualmente diversos campos conducen investigaciones sobre el dominio "género". Este estudio investiga bajo qué perspectivas este dominio es abordado por la Ciencia de la Información (Cl). Objetivo: El objetivo es identificar cómo el campo se apropia y/o contribuye a los estudios sobre género, con la acepción de que género es un término relacionado con los roles sociales y expectativas de comportamiento atribuidos a las personas en función de su sexo. Metodología: Se realizó la recolección y análisis de las palabras clave de 588 artículos indexados en International Library and Information Science Abstracts (LISA), entre 1980 y 2016. Considerando el gran número de palabras clave recuperadas, se generó una nube de

Inf. Inf., Londrina, v. 22, n. 2, p. 144 - 165, maio/ago., 2017.

http:www.uel.br/revistas/informacao/ 
tags; permitiendo la identificación de los términos más utilizados por década. Resultados: Los resultados indican que el dominio "género" fue abordado por la $\mathrm{Cl}$ bajo diferentes perspectivas en los últimos años, pero manteniendo, en general, el foco principal en cuestiones relacionadas a estudios sobre mujeres y diferencias de género. Además, se observa que el dominio es tratado por la $\mathrm{Cl}$ principalmente dentro de tópicos tradicionales del área, como Recuperación de la Información y Comunicación Científica. Conclusiones: Se resalta que el panorama resultante de este estudio abre nuevas posibilidades de investigación sobre cómo la $\mathrm{Cl}$ aborda el dominio "género" en sus estudios científicos.

Palabras clave: Género. Ciencia de la Información. Estado de Conocimiento.

Recebido: 10.03.2017

Aceito: 25.08 .2017

Inf. Inf., Londrina, v. 22, n. 2, p. 144 - 165, maio/ago., 2017.

http:www.uel.br/revistas/informacao/ 
Mell Siciliano, Cleiton da Mota de Souza, Clara de Mello e Souza Meth Sobre o que falamos quando falamos em gênero na ciência da informação?

APÊNDICE A - Quadro de palavras-chave dos anos 1980

\begin{tabular}{|l|c|}
\hline \multicolumn{1}{|c|}{ Palavras-Chave } & Frequência \\
\hline Gender & 3 \\
\hline Efficiency; Evaluation; Job performance; Library staff & 2 \\
\hline $\begin{array}{l}\text { Children; Information work; Leisure; Libraries; Library } \\
\text { materials; Public libraries; Reading; Reference work; } \\
\text { Sexism; Sexual behaviour; Special libraries; Use }\end{array}$ & 1 \\
\hline
\end{tabular}

Inf. Inf., Londrina, v. 22, n. 2, p. 144 - 165, maio/ago., 2017. 
Mell Siciliano, Cleiton da Mota de Souza, Clara de Mello e Souza Meth

Sobre o que falamos quando falamos em gênero na ciência da informação?

APÊNDICE B - Quadro de palavras-chave dos anos 1990

\begin{tabular}{|c|c|}
\hline Palavra-Chave & Frequência \\
\hline Gender & 59 \\
\hline Information & 12 \\
\hline Communication; Women & 11 \\
\hline World Wide Web & 10 \\
\hline Library staff & 8 \\
\hline Children; Gender differences; Internet; Publishing; Users & 7 \\
\hline Discussion groups; Information technology & 6 \\
\hline Chatrooms; Education; Research; Use & 5 \\
\hline $\begin{array}{l}\text { Communications technology; Fiction; Information seeking behaviour; } \\
\text { Libraries; Men }\end{array}$ & 4 \\
\hline $\begin{array}{l}\text { Computers; Curricula; Feminism; Gender equity; Librarianship; Literature; } \\
\text { Professional education; School resource centres; Technical writing; } \\
\text { Young people }\end{array}$ & 3 \\
\hline $\begin{array}{l}\text { Computer literacy; Coverage; Gender training; History; information } \\
\text { retrieval; Library materials; Massmedia; Newspapers; Publications; } \\
\text { Reference work; Science; Secondary schools; Sexual discrimination; } \\
\text { Social aspects; Subject indexing; Technology; Websites }\end{array}$ & 2 \\
\hline $\begin{array}{l}\text { Advertising; Alcohol; Antiquarian materials; Archives; Author productivity; } \\
\text { Authors; Autobiography; Avatars; Biological aspects; Books; Boys; Career } \\
\text { development; Chat; Childhood; Chinese materials; Class; Cognitive; } \\
\text { Computer; Computer games; Cultural aspects; Design; Educational } \\
\text { technology; Election campaigns; Electronic commerce; Erotic } \\
\text { publications; Ethnic groups; Ethnic studies; Gender issues; Gender } \\
\text { neutrality; Gender studies; Girls; Indexing; Information centres; } \\
\text { Information services; Information society; Information storage; Information } \\
\text { work; International organizations; Journalists; Knowledge; Leadership; } \\
\text { Learning; Library associations; Library managers; Library schools; Library } \\
\text { technology; Literature; Magazines; Multiple Worlds; Multiuser domains; } \\
\text { Music; Musicology; National information infrastructure; National policies; } \\
\text { News; Nonfiction; Online; Oral communication; Organizations; Parents; } \\
\text { Persuasion; Pornography; Postgraduate students; Pseudonyms; Public } \\
\text { libraries; Qualifications; Query formulation; Reading; Reference materials; } \\
\text { Reluctant readers; Research Libraries; Rooms; Salaries; Sales; Schools; } \\
\text { Searching; Sexual harassment; Social issues; Status; Students; Style; } \\
\text { Teachers; Teaching; Teaching methods; Technological universities; } \\
\text { Telecommuting; Theories; Tropical agriculture; Universities; University } \\
\text { libraries; Violence; Virtual communities; Virtual reality; Women studies; } \\
\text { World War } 2\end{array}$ & 1 \\
\hline
\end{tabular}

Inf. Inf., Londrina, v. 22, n. 2, p. 144 - 165, maio/ago., 2017.

http:www.uel.br/revistas/informacao/ 
Mell Siciliano, Cleiton da Mota de Souza, Clara de Mello e Souza Meth

Sobre o que falamos quando falamos em gênero na ciência da informação?

APÊNDICE C - Quadro de palavras-chave dos anos 2000

\begin{tabular}{|c|c|}
\hline Palavra-Chave & Frequência \\
\hline Gender & 117 \\
\hline Gender differences & 51 \\
\hline Women & 29 \\
\hline Information technology & 28 \\
\hline Users & 27 \\
\hline Internet & 21 \\
\hline Gender equity & 18 \\
\hline User & 16 \\
\hline Online & 15 \\
\hline Children; Information retrieval; World Wide Web & 13 \\
\hline Education; Research & 12 \\
\hline Publishing & 10 \\
\hline Searching; Social aspects; Use & 9 \\
\hline $\begin{array}{l}\text { Attitudes; Bibliometrics; Communications technology; Computers; Distance } \\
\text { learning; Feminism; Information; Information seeking behaviour; Libraries; } \\
\text { Mass media; Reading; Women studies; }\end{array}$ & 8 \\
\hline Books; Boys; Students & 7 \\
\hline Girls; Google; Scholarly publishing; Websites & 6 \\
\hline $\begin{array}{l}\text { Author productivity; Educational technology; Ethnic groups; Human computer } \\
\text { interaction; Information science; Library staff; Newspapers; Periodicals; } \\
\text { Public libraries; Salaries; Scientometrics; Stereotypes; Television; User } \\
\text { behaviour; Young people }\end{array}$ & 5 \\
\hline $\begin{array}{l}\text { Access; Articles; Authors; Computer applications; Computer literacy; Content } \\
\text { analysis; Ethics; Evaluation; Information; Information professionals; } \\
\text { Language; Media; Men; Relationship; Secondary schools; Universities }\end{array}$ & 4 \\
\hline $\begin{array}{l}\text { Academic libraries; Advertising; Aspects; Bibles; Career choice; Children; } \\
\text { Computer industry; Computer mediated communication; Computer science; } \\
\text { Cultural aspects; Democracy; Discussion groups; Fiction; History; } \\
\text { Information literacy; Interface; Journalism; Library science; Public; Sexual } \\
\text { discrimination; Social; Software; Status; Translations; University libraries }\end{array}$ & 3 \\
\hline $\begin{array}{l}\text { Agriculture; Anxiety; Bias; Bibliographies; Business; Career development; } \\
\text { Chief librarians; Citizenship; Computer skills; Controlled vocabulary; Digital; } \\
\text { Electronic commerce; Electronic information services; Electronic mail; } \\
\text { Electronic publishing; Government information; Health; Higher education; } \\
\text { Information communication; Law; Library management; Mental health; } \\
\text { Natural resources; Online data bases; Open universities; Portrayal; } \\
\text { Promotion; Racial discrimination; Radio; Researchers; Role; Rural areas; } \\
\text { School children; Science; Scientists; Sex; Spanish language; Speech; }\end{array}$ & 2 \\
\hline
\end{tabular}

Inf. Inf., Londrina, v. 22, n. 2, p. 144 - 165, maio/ago., 2017.

http:www.uel.br/revistas/informacao/ 
Mell Siciliano, Cleiton da Mota de Souza, Clara de Mello e Souza Meth

Sobre o que falamos quando falamos em gênero na ciência da informação?

Teachers; Terminology; User interface; Young people; Advertisements

Academic achievement; Access to information; Age; Analysis; Applications;

Archives; Biotechnology; Blogs; Broadcasting; Business management;

Careers; Cataloguing; Chemistry; Chief; Childrens books; Christianity;

Citations; Classification; Coauthorship; Collaboration; Communication;

Computer games; Conferences; Consultants; Cooperative learning;

Credibility; Critical research; Cultural; Culture; Depression; Design;

Developing countries; Dialect; Dictionaries; Differences; Directors; Disadvantaged people; Discourse analysis; Doctorates; Election campaigns; Elections; Electronic; Electronic books; Electronic government; Electronic media; Electronic money; Electronic resources; Employees; Employment; Employment opportunities; Epistemology; Equal pay; Equity; Ethnic; Executives; Experience; Experts; Faculty; Families; Fidelity; Free lance staff; Full text databases; Gender Coverage; Gender morphing; Gender studies; Gender Watch; Geography; God; Heroes; Homosexuality; Identity; Illustrations; Immigrants; Impact factors; Indexing

Information industry; Information management; Information services; Information systems; Input; Interaction; Interviews; Job; Journalists; Knowledge; Knowledge acquisition; Knowledge economy; Knowledge representation; Learning; Lexicons; Librarians; Librarianship; Library managers; Linguistics; Links; Literacy; Literature; Loyalty; Machine; Magazines; Man; Managers; Media; Mediation; Medicine; Membership; Mobile communications; Motivation; Music; Networks; News services; Novices; Olympic games; Online catalogues; Online payment; Organizational culture; Organizational learning; Patents; Peer review; Performance; Photographs; Picturebooks; Political aspects; Programming; Psychological aspects; Psychology; Publication; Public Libraries; Questionnaires; Race; Readers; Reference; Relevance; Reporters; Reporting; Research libraries; Resource centres; Retrieval; Rock; Satisfaction; Scholarly publications; School; School librarians; School libraries; Search engines; Seeking behaviour; Selection; Sexuality; Shopping; Shows; Social class; Social exclusion; Social identity; Social networks; Social sciences; Speech recognition

Staff; State government; Statistical methods; Statistics; Status; Stress; Studies; Style; Systems development; Tagging; Talk; Teaching; Teamwork; Technology; Teleworking; Text messaging; Theories; Theses; Transactions; Usability; Users; User services; User training; Video; Video conferencing; Viewers; Virtual communities; War; Web databases; Web of Science; Webometrics; Wireless communications; Work; Working conditions

Inf. Inf., Londrina, v. 22, n. 2, p. 144 - 165, maio/ago., 2017. 
Mell Siciliano, Cleiton da Mota de Souza, Clara de Mello e Souza Meth

Sobre o que falamos quando falamos em gênero na ciência da informação?

APÊNDICE D - Quadro de palavras-chave dos anos 2010

\begin{tabular}{|c|c|}
\hline Palavras-Chave & Frequência \\
\hline Gender & 145 \\
\hline Gender differences & 55 \\
\hline Women & 34 \\
\hline Social networks & 29 \\
\hline Scholarly publishing & 23 \\
\hline Internet; Students & 16 \\
\hline Research & 15 \\
\hline Information technology & 14 \\
\hline Librarians; Researchers & 13 \\
\hline Academic libraries; Communication & 11 \\
\hline Bibliometrics; Gender equity; Publication output; Websites; Young people & 12 \\
\hline User behaviour & 10 \\
\hline $\begin{array}{l}\text { Author productivity; Females; Information seeking behaviour; Information } \\
\text { sources; Use }\end{array}$ & 9 \\
\hline Blogs; Computers; Information; Perceptions; Social aspects & 8 \\
\hline $\begin{array}{l}\text { Attitudes; Citation analysis; Coauthorship; Education; Electronic media; } \\
\text { Higher education; Science; Studies }\end{array}$ & 7 \\
\hline $\begin{array}{l}\text { Communications technology; Digital divide; Distance learning; Electronic } \\
\text { commerce; Medicine; Profession; Sexuality; Technology; Universities }\end{array}$ & 6 \\
\hline $\begin{array}{l}\text { Age factors; Authorship; Cellular telephones; Demography; Design; } \\
\text { Emotions; Feminism; Information communication; Information systems; } \\
\text { Learning; Motivation }\end{array}$ & 5 \\
\hline $\begin{array}{l}\text { Career development; Collaboration; College students; Consumer health } \\
\text { information; Cultural aspects; Ethics; Faculty; Health education; Health } \\
\text { promotion; International comparisons; Law; Legal research; Online shopping; } \\
\text { Privacy; Reading; Scholarly communication; Social exclusion; Stereotypes; } \\
\text { University libraries; Virtual communities }\end{array}$ & 4 \\
\hline $\begin{array}{l}\text { Age Analysis; Authors; Behavior; Bias; Books; Candidates; Citations; } \\
\text { Comparisons; Computer science; Computer skills; Employees; Ethnic } \\
\text { groups; Games; Girls; Hypotheses; Impact analysis; Interventions; } \\
\text { Language; Librarianship; Literacy; Peer review; Race; Salaries; } \\
\text { Scientometrics; Search engines; Social research; Software engineering; } \\
\text { Undergraduate students; Usability; Users; Womens studies }\end{array}$ & 3 \\
\hline $\begin{array}{l}\text { Academic achievement; Academic status; Advertising; Anxiety; Articles; } \\
\text { Assessment; Biometrics; Branding; Cancer; Censorship; Chemistry; } \\
\text { Cognitive style; } \\
\text { Colleges; Computer based; Computer games; Content analysis; } \\
\text { Cooperation; Culture; Diagnosis; Digital libraries; Doctors; Economics; } \\
\text { Efficacy; Elections; Electronic books; Electronic government; }\end{array}$ & 2 \\
\hline
\end{tabular}

Inf. Inf., Londrina, v. 22, n. 2, p. 144 - 165, maio/ago., 2017.

http:www.uel.br/revistas/informacao/ 
Encyclopaedias; Enrollment; Evaluation; Feasibility; Funding; Gender identity; Health; Health behaviour; Healthcare; Health information; Health status; History; Homosexuality; Human rights; Identification; Impact factors; Information literacy; Information retrieval; Information seeking; International relations; Knowledge management; Library cataloguing; Library history; Library science; Life sciences; Literature reviews; Males; Medical research; Men; Multiculturalism; Multimedia; Nanotechnology; Negative campaigning; Newspapers; Participation; Partisanship; Personality; Physical activity; Picture books; Predictions; Primary schools; Printed materials; Psychology; Public libraries; Quality; Reform; Research centers; Role models; Sampling; Scientists; Secondary school; Sexual discrimination; Social capital; Social sciences;

Software; Special libraries; Teaching; Technology adoption; Television; Text analysis; Text books; Theses; Transgendered people; Usefulness; Videogames

Aborigines; Academic publications; Academic staff; Access; Accuracy; Adolescents; Advertisements; Age differences; AIDS; Alcohol consumption; Alcohol use; Anonymity; Application; Archaeology; Auctions; Barriers; Bibliographic databases; Bibliographies; Biology; Black women; Boards; Body mass; Book reviews; Brand image; Breast cancer; Browsing; Campaigns; Career choice; Chemical engineering; Children; Childrens books; Childrens literature; Citation indexes; Citizens; Classification; Clinical information; Clustering; Cognitive aspects; Collection development; Colour; Community; Comprehension; Computerforensics; Computer industry; Confidentiality; Congressional elections; Consciousness; Content management software; Context; Copyright; Courtcases; Credibility; Critical thinking; Curricula; Customization; CVs; Datacollection; Datamining; Data security; Data sets; Data warehouses; Departments; Development; Diabetes; Disabled people; Disadvantaged people; Discourse analysis; Disease control; Disease management; Disease prevention; Distribution; Doctorates; Domestic violence; Ebooks; Editing; Editorial policies; Editors; Educational psychology; Educational technology; Education libraries; Elderly people; Election results; Electronic journals; Electronic newspapers; Engineering; Ethnic foods; Ethnic group; Ethnography; Facebook; Fiction; Food; Food Preparation; Foreign students; Gender access; Gender studies; General practice; General practitioners; Globalization; Government information; Graduate students; Grant applications; Graphs; Grey literature; Guidelines; Guides; Health costs; Health disparities; Health policy; Health professionals; Health services; Helpseeking; Highschool; HIV; Homemakers; Human aspects; Hypermedia; Ideology; Imbalance; Income; Infections; Informal education; Information centres; Information professionals; Information work; Instant messaging; Interactive learning; Interlibrary loans; Investment; Job hunting; Job satisfaction; Journalists; Journals; Keywords; Knowledge; Knowledge organization; Labour; Leadership; Learning management systems; Legislative Bodies; Leisure; Libraries; Library materials; Loyalty; Managers; Marketing; Market research; Market strategy; Masculinity; Mathematical models; Measurement; Medical informatics; Medical school libraries; Medical treatment; Mens health; MeSH; Meta analysis; Methodology; Methods; Minority groups; Modification; Music; National policies; Natural language processing; Navigation; Networking; Nurses; Obesity; Old people; Online; Online databases; Online information retrieval; Open access; Opensource; Opposite Sex Relations; Organizational culture; Out reach services; Parents; Passive smoking; Patent applications; Patents; Path analysis Patients; Pattern recognition; Periodicals; Personal information; Personality traits; Philosophy; Photographs; Piracy; Pluralism; Political; Political advertising; Political aspects; Political behavior; Political 
Campaigns; Political representation; Politics; Popular culture; Portable computers; Postgraduate students; Presidential Campaigns; Private schools; Problem solving; Process evaluation; Productivity; Product reviews; Professional responsibilities; Professors; Profiles; Promotion; Psychiatry; Psychological aspects; Qualifications; Readers; Recruitment; Reliability; Religion; Retention; Reviews; Rockmusic; Rural areas; School; School libraries; Searching; Search strategies; Security management; Selection; Self efficacy; Self image; Self management; Semantics; Sex; Sex discrimination; Sex Roles; Sites; Skills; Smartphones; Social change; Social motivation; Social responsibility; Social scientists; Society; Socio demographic aspects; Socioeconomic factors; Socioeconomic status; Source materials; Speakers; Sports; Standards; Statistics; Status; Student participation; Subject fields; Symptoms; Tables; Tagging; Teachers; Technical education; Technology acceptance; Teenagers; Theories; Theory; Thesauri; Timeuse; Tobacco; Trends; Trust; Turkey; Uncertainty; Uptake; Urban areas; User training; Variables; Video; Violence; Virtual teams; Web; Web browsers; Weight; Wikis; Womens health; Working conditions; Worklife balance; World Wide Web; Writers; Writing 